\title{
FAMILIA MONOMARENTAL Y RIESGO DE EXCLUSIÓN SOCIAL ${ }^{1}$
}

\section{MONOMARENTAL FAMILY AND RISK OF SOCIAL EXCLUSION}

\author{
Rosa Santibáñez. Universidad de Deusto - España \\ rosa.santibanez@deusto.es \\ Natalia Flores. Universidad de Deusto - España \\ nataliaflores@deusto.es \\ Alba Martín. Universidad de Deusto - España \\ alba.martin@opendeusto.es
}

\begin{abstract}
Resumen La vida familiar se ha enfrentado a profundos cambios durante las últimas décadas experimentando transformaciones a nivel estructural, valorativo, actitudinal y funcional. En años recientes, especialmente a raíz de la crisis económica de 2008 , el debate sobre la creciente desigualdad socioeconómica del mundo ha cobrado fuerza. Este debate se ha visto reforzado no sólo por concebir la pobreza desde una perspectiva económica sino también desde la social y de exclusión. Existen muy pocos estudios realizados en torno al tema de la monoparentalidad y la monomarentalidad. Este artículo se plantea con tres objetivos: Describir la evolución de los conceptos monoparental y monomarental, analizar su realidad y sus necesidades. Y finalmente, examinar la vinculación de la monomarentalidad con la exclusión social. La revisión constata la realidad creciente de estas familias junto a las necesidades vinculadas a las mismas y el riesgo de exclusión.
\end{abstract}

Palabras clave Monomarental, monoparental, riesgo de exclusión social, familia.

\begin{abstract}
Family life has faced profound changes over recent decades, experiencing structural, evaluative, attitudinal and functional transformations. In recent years, especially as a result of the 2008 economic crisis, the debate over the growing socioeconomic inequality of the world has gained strength. This debate has been reinforced not only by conceiving poverty from an economic perspective but also from a social point of view of and as regards exclusion. There are very few studies on the issue of single parenthood and single motherhood. This article is presented with three objectives: to describe the evolution of the concepts single-parent and single-mother (Monoparental and Monomarental), to analyse their reality and their needs, and finally, to examine the link between single motherhood and social exclusion. The review notes the growing reality of these families together with the needs linked to them and the risk of exclusion.
\end{abstract}

Keywords Monomarental, single parent, risk of social exclusion, family.

\footnotetext{
${ }^{1}$ El presente artículo forma parte de un estudio más amplio realizado desde la Universidad de Deusto para la Diputación Foral de Bizkaia dentro del acuerdo Bizkailab (Santibáñez, Flores y Martín, 2017). This article is part of a larger study carried out by the University of Deusto for the Provincial Council of Bizkaia within the Bizkailab agreement (Santibáñez, Flores and Martín, 2017).
}

Orcid: $0000-0002-6260-1130$

Orcid: $0000-0003-0135-3839$

Orcid: 0000-0001-9151-328X

Recibido: 23.10.2017

Aceptado: 16.11.2017 


\section{Introducción}

La vida familiar se ha enfrentado a profundos cambios durante las últimas décadas experimentando transformaciones a nivel estructural, valorativo, actitudinal y funcional. Los factores del cambio han sido de naturaleza muy variada y al mismo tiempo transversal. El declive de la fecundidad, el aplazamiento o desistimiento del matrimonio, el aumento de las tasas de separación, la incorporación de las mujeres al mercado laboral, la interrupción voluntaria del embarazo y el cambio de roles, entre otros, son algunos de los cambios que han causado dichas transformaciones, dando lugar entre otras tipologías a las familias monoparentales y monomarentales (Alter, 2008; Fundación Foessa, 2008; Perondi, 2012).

Ciertamente, este tipo de familia -la familia monoparental- pese a la idea que se ha tratado de transmitir de una única realidad familiar, la nuclear convencional, es un modelo familiar que ha existido históricamente. No obstante, ha estado sometida a estereotipos como "familia desviada" infravalorando perenemente su labor en la crianza de los hijos (Alter, 2008; Avilés, 2012). Los datos ofrecidos por Save The Children (Sastre, 2015) advierten que, las familias monoparentales, en su inmensa mayoría están formadas por una mujer sola a cargo de su prole ( $82 \%$ de los casos). Asimismo, este escenario familiar se correlaciona frecuentemente con la pobreza o la exclusión social, afectando a más de la mitad de los hogares monomarentales (53,3\%).

En años recientes, especialmente a raíz de la crisis económica de 2008 , el debate sobre la creciente desigualdad socioeconómica del mundo ha cobrado fuerza. Este debate se ha visto reforzado no sólo por concebir la pobreza desde una perspectiva económica sino también desde la social y de exclusión. Los países en los que la desigualdad ha aumentado, registran también una mayor aceleración de inestabilidad social, perjudicando en mayor medida a la infancia que vive en hogares empobrecidos -a menudo en familias monomarentales-. Debido a las consecuencias de la desigualdad y la dificultad que tienen de modificar estas familias las oportunidades y/o estilos de vida, a menudo están sujetas a decisiones y elecciones de su entorno familiar y, su futuro estatus dependerá del de sus padres y/o madres: "No solo hay más pobreza, sino que ésta se hereda en mayor medida" (Sastre y Escorial, 2017, p.5).

Existen muy pocos estudios realizados en torno al tema de la monoparentalidad y la monomarentalidad (entendiendo que el primer término ha sido utilizado para referirse tanto a los casos de familias formadas por una madre como por un padre). En este escenario el siguiente artículo se plantea con tres objetivos: En primer lugar, describir la evolución del concepto Monoparentalidad y sus significados e implicaciones hasta la aparición del término Monomarentalidad. En segundo lugar, analizar la realidad de las familias monomarentales y sus necesidades en función de las diferentes vías de acceso a esta condición. Y finalmente, en tercer lugar, examinar la vinculación de la monomarentalidad con la exclusión social y sus consecuencias en relación a la transmisión intergeneracional de la pobreza. 
Desde la perspectiva metodológica y para el cumplimiento de estos tres objetivos se ha realizado una revisión bibliográfica sistemática a nivel internacional y estatal. La búsqueda bibliográfica parte de un componente exploratorio de los términos: familia monomarental y exclusión social en las bases de datos internacionales ERIC (Education Resources Information Center) y Psyclnfo (American Psychological Association), así como enlas fuentes más cercanas como son el SIIS $^{2}$ y los observatorios o centros de estudio como el Instituto de la Mujer, Emakunde ${ }^{3}$, Ararteko ${ }^{4}$ e Ikuspegi ${ }^{5}$. Los materiales más específicos localizados, se organizan en torno a los ejes temáticos de este artículo: la evolución de los conceptos monoparental y monomarental, la evolución sociológica de estas familias en el contexto europeo y español, así como su vinculación con estados de riesgo, pobreza o necesidad.

La estructura del artículo refleja la consecución de los objetivos de manera general. Sin embargo, sí resulta importante aclarar que el uso, casi alternativo de los términos monoparental / monomarental, no es el fruto de una incoherencia sino más bien el reflejo de la complejidad y el estado de la cuestión. Es la complejidad que vertebra esta temática lo que da fruto a su elección diferente en cada apartado. Además de existir un número muy escaso de investigaciones, tal y como hemos mencionado anteriormente, la cifra se reduce aún más si colocamos el foco de estudio en la cuestión "monomarental". Es por ello que cuando hemos querido hacer alusión de forma concreta a este modelo familiar, hemos utilizado dicho término para reivindicar la especificidad de su significado en un contexto de escasez de datos, de donde hemos querido rescatar en ese aparente juego de palabras (monopa-monoma) una realidad invisibilizada. Para aludir a su frágil situación (cuando la vía de acceso es "no elegida") y plantear la necesidad de co-crear respuestas y alternativas que proporcionen calidad de vida a las personas que conforman todos los modelos de familia existentes.

\section{La familia monoparental - monomarental: ¿Un nuevo modelo?}

Con los procesos de industrialización y los cambios sociales colindantes, la familia nuclear biparental, se fue haciendo cada vez más presente hasta el punto de convertirse en la forma familiar dominante y el modelo ideal de la sociedad moderna. Este hecho tuvo importantes implicaciones para todas aquellas familias que

\footnotetext{
${ }^{2}$ Centro de documentación y Estudios sobre Servicios Sociales y Política Social de la Fundación Eguia Careaga. http://www.siis.net/

${ }^{3}$ Instituto Vasco de la Mujer. http://www.emakunde.euskadi.eus/inicio/

${ }^{4}$ Defensor del Pueblo del País Vasco. http://www.ararteko.net/

5 Observatorio de la Familia en el País Vasco. http://www.euskadi.eus/webo1a2famil/es/contenidos/informacion/publicaciones_observatorios/es_publica/index.shtml
} 
presentaban, por diversos motivos, una estructura diferente a dicho modelo y comenzaron a ser calificadas como disfuncionales ya que, en teoría, no presentaban los requisitos necesarios para el desempeño correcto de la crianza de la prole. Como consecuencia directa, la mayoría de éstas se convirtieron en objeto de rechazo y exclusión social (Alter, 2008).

No es hasta finales del siglo XX cuando se produce un claro cambio en las estructuras familiares. Las trayectorias de vida y las relaciones intrafamiliares se encuentran inmersas en un proceso de profunda transformación, adaptándose a las múltiples necesidades de nuestra sociedad (Castro y Seiz, 2014). El declive de la fecundidad, el aplazamiento o la ausencia del matrimonio, el aumento de las tasas de separación, la incorporación de las mujeres al mundo laboral y la interrupción voluntaria del embarazo son, entre otros, cambios que rompen con el modelo normativo de la familia nuclear convencional (Castro y Seiz, 2014). Tal y como afirma UNICEF (2003), en tan sólo treinta años hemos pasado de una época en la que la mayoría de las parejas se casaban, a otra, en la que la mayoría demora su edad de contraer matrimonio y se observa una creciente minoría que nunca se casará. El Instituto Nacional de Estadística - INE enfatiza que en 2015 el número de parejas de hecho aumentó un 1,6\%, mientras que el de parejas casadas descendió un 2,2\% en comparación con los datos del 2014, situándose así en un 14,5\% las parejas de hecho, frente a un $85,5 \%$ las parejas casadas (INE, 2016).

Esta diversidad familiar, entendida como quiebra del modelo tradicional de la familia nuclear convencional, ha implicado un principio de desinstitucionalización y privatización de las relaciones familiares, dando lugar a nuevos modelos familiares que pueden alterar su funcionamiento y el ajuste psicosocial de sus integrantes. Desde esta perspectiva, Andolfi (1993, p.27) define la familia como el "conjunto organizado e interdependiente de personas en constante interacción, que se regula por unas reglas y por funciones dinámicas que existen entre sí y con el exterior".

De Lourdes (2003) examina esta pluralidad de familias, muchas ya consolidadas, otras en ascenso y algunas, todavía como modelos emergentes: la familia nuclear convencional $^{6}$, la familia extensa ${ }^{7}$, la familia reconstituida, polinuclear o mosaico ${ }^{8}$ y la familia monoparental/ monomarental a la que dedicamos este artículo.

A esta tipología familiar, Lorence, Pérez, Menéndez y Rodríguez (2015), MartínezMonteagudo (2013) y Castro y Seiz (2014) añaden, en estudios más recientes, nuevas

\footnotetext{
${ }^{6}$ Es el modelo de familia tradicional, que implica la presencia de un hombre y una mujer con los/as hijos/as en común, viviendo todos bajo el mismo techo.

${ }^{7}$ La familia extensa está constituida por el tronco familiar principal (padres, hijos y/o abuelos) al que se le unen otras personas que proceden de ramas colaterales.

${ }^{8}$ Familias en la que al menos un miembro de la pareja proviene de una unión anterior. Tras la separación, el divorcio o la viudedad, son los progenitores que vuelven a formar una pareja con otros para iniciar una nueva relación. Valdivia Sánchez (2008), añade que también son conocidas como "bifocales" o "multiparentales".
} 
formas y estructuras familiares, señalando las siguientes: la familia homoparental ${ }^{9}$, la familia nuclear simple ${ }^{10}$, la familia polinuclear ${ }^{11} \mathrm{y}$, finalmente, la familia transnacional ${ }^{12}$. Sirvan estas clasificaciones tan sólo como ejemplo de lo que acabamos de introducir.

Si la industrialización trajo asociado un único modelo familiar como viable y útil, el final del siglo XX y el principio del XXI se han manifestado como una eclosión de la diversidad de estructuras y funcionamientos. Es por ello, que esta evolución creciente de la familia, hace que actualmente nos encontremos con una gran diversidad de tipologías familiares, que, si bien comparten necesidades comunes, también encuentran otras de mayor especificidad.

Las familias monoparentales y monomarentales están constituidas por un padre o una madre a cargo de al menos un hijo o hija menor de 18 años. La definición operativa utilizada en los estudios sociológicos (Perondi, 2012, p. 38) es la siguiente:

"Se consideran familias monoparentales o monomarentales, a efectos de este estudio, los núcleos familiares constituidos por una sola persona adulta, ya sea hombre o mujer, y al menos una persona menor. Se entiende por persona menor a aquella residente en el hogar, que tiene menos de 18 años y a aquella de entre 18 y 24 años (inclusive) que no desarrolla actividad remunerada $y$, por tanto, no aporta ingresos laborales al mismo."

No es hasta finales del siglo XX, cuando en España se ha ido ampliando el estudio sobre las familias monoparentales $y$, a principios del siglo XXI cuando se recategoriza a estas familias en términos femeninos, bajo el nombre de familias monomarentales, al observar que la mayoría de estas familias están representadas por la figura de la madre como cuidadora de sus hijos/as. Esta necesidad de reconceptualizar la realidad familiar pretende alzar la capacidad de la figura de las mujeres en la crianza de su prole, evitar el estigma social de las denominaciones anteriores y aumentar el número de estudios sobre estas familias. Asimismo, España ha sido uno de los países de su entorno que más ha tardado en explicitar este reconocimiento, debido a la situación sociopolítica y religiosa protagonizada con la época franquista (Song, 1996).

La búsqueda en los tesauros de las bases de datos internacionales, permite además de localizar material bibliográfico relevante, explorar la representación mental del mundo científico del momento. En otras palabras, si un término es

\footnotetext{
${ }^{9}$ Constituidas por parejas del mismo sexo: dos hombres o dos mujeres. España ha sido el tercer país europeo, tras Holanda y Bélgica que ha reconocido el matrimonio legal entre dos personas del mismo sexo. Lo hizo en el año 2005.

${ }^{10}$ Formadas por una pareja sin hijos/as.

${ }^{11}$ Padres o madres que deben atender económicamente, además de a su actual pareja, algún hogar abandonado tras el divorcio o la separación.

${ }^{12}$ Familias estables que por la movilidad de uno o ambos miembros de la familia pasan temporadas separadas residiendo en distintos países.
} 
reconocido o no en una base de datos internacional es un indicador o una evidencia de que se está desarrollando un volumen importante de investigación en la materia y por ello, se hace necesaria su incorporación para la clasificación y tratamiento de dicha información. Es por ello, que se analiza la aparición o no de los términos o descriptores utilizados por las dos bases de datos más importantes internacionales en educación y psicología. De aparecer, se añade en qué año fueron incorporados y las definiciones acordadas. La siguiente tabla recoge los resultados encontrados en materia de terminología utilizada en este ámbito.

Tabla 1: Términos encontrados en los tesauros ERIC y Psyclnfo

\begin{tabular}{|c|c|c|c|}
\hline ÁREA & DESCRIPTOR & AÑo & DEFINICIÓN \\
\hline \multirow{3}{*}{$\begin{array}{l}\text { Familiar } \\
\text { structure }\end{array}$} & Single parents & 1978 & $\begin{array}{l}\text { Padres que crían en soledad a } \\
\text { sus hijos/as }\end{array}$ \\
\hline & Single mother & 1994 & - \\
\hline & Unwed mothers & 1973 & $\begin{array}{l}\text { Incluye también a madres } \\
\text { adolescentes }\end{array}$ \\
\hline \multirow{3}{*}{$\begin{array}{l}\text { Civil } \\
\text { status }\end{array}$} & Divorced persons & 1973 & - \\
\hline & Divorced* & - & $\begin{array}{l}\text { Disolución legal del } \\
\text { matrimonio }\end{array}$ \\
\hline & $\begin{array}{l}\text { Marital } \\
\text { separation }\end{array}$ & 1973 & - \\
\hline \multirow{4}{*}{$\begin{array}{l}\text { Economi } \\
\text { c } \\
\text { situation }\end{array}$} & Poverty areas & 1973 & Suburbios \\
\hline & Poverty areas* & - & $\begin{array}{l}\text { Zonas geográficas } \\
\text { caracterizas por privación } \\
\text { económica }\end{array}$ \\
\hline & Poverty & 1973 & $\begin{array}{l}\text { Falta de medios para adquirir } \\
\text { material necesario o } \\
\text { comodidades }\end{array}$ \\
\hline & Low income level & 1973 & - \\
\hline
\end{tabular}

Fuente: Elaboración propia. (*ERIC, resto Psyclnfo)

Algunos comentarios que se derivan de esta tabla 1 son los siguientes: En primer lugar, es importante destacar que prácticamente la totalidad de los términos han sido incluidos en los años 70. Tan sólo uno de ellos, single mother (madre soltera), se incorpora en los años 9o. Dentro del apartado de estructura familiar se recogen en 1973 términos como unwed mother (madre soltera pero incluye a adolescentes) y parental absence (ausencia parental), para describir la ausencia de uno de los dos cónyuges en la familia. En 1978 Pyclnfo recoge la palabra single parent (familia monoparental sin especificar género) y 16 años después, incorpora -sin mostrar su definición- el término single mothers, que es más específico para referirse a "la madre 
sola". En referencia al estado civil y siempre haciendo referencia a una situación jurídica, más que social, se encuentran términos conexos con el divorcio y la separación matrimonial, también en la década de los 70, sin aportar más información o avances en las temáticas. Haciendo alusión al área que describe la situación económica, se puede observar nuevamente que 1973 es un año trascendental para definir los ejes principales de la investigación. Algunos de los términos más tradicionales se encuentran sustentados con una definición como la de pobreza. Sin embargo, otros conceptos como familias pobres o la transmisión de la pobreza no se encuentran aún recogidos y mucho menos los más específicos (necesidades de la familia monoparental, desigualdades y exclusión social, trasmisión intergeneracional de la pobreza...), por lo que se manifiesta como un ámbito aún sin explorar que debe ser estudiado para poder acceder con más facilidad a la realidad de estas familias. En suma, la evolución de los términos en los tesauros internacionales de psicología y educación, entre los años 70 y 90 ha supuesto un avance únicamente en lo referido a la estructura familiar y su situación civil y económica.

En nuestro entorno más cercano, destaca un estudio realizado por Rodríguez y Luengo (2003, p.66) en donde analizan un total de nueve definiciones que aparecen en las décadas de los 80 y 90, que ponen de manifiesto cómo de las 9 definiciones encontradas y analizadas, incluida una de la Comisión Europea, tan sólo 4 de ellas, reconocen el sexo / género del progenitor como variable clave de la unidad familiar. Sin embargo, parece tratarse de una de las variables más importantes en la caracterización de los hogares monomarentales y más específicamente de los que se encuentran en riesgo de pobreza o en situación de pobreza.

\section{Analizando las vías de entrada a la monomarentalidad}

Aunque el término conocido actualmente como familias monomarentales es ciertamente novedoso, su presencia sociológica ha sido una constante a lo largo de la historia. Prueba de ello es que, como destacan algunas investigaciones (Avilés, 2012; 2015i Barrón, 2002, Samaranch y Di Nella, 2011), siempre han existido las procreaciones fuera del matrimonio, las separaciones entre los cónyuges por motivos sociales (encarcelamiento, guerra, migraciones...) o la viudedad. Pese a las duras críticas que algunas de estas fórmulas han suscitado, no todas estas familias han sido consideradas de la misma manera, sino que han existido importantes diferencias sociales y morales en función de la causa de la ruptura.

A finales del siglo XX, Miri Song (1996) investiga en Inglaterra las ayudas económicas que ofrecía el estado para éstas, llegando a la conclusión de que las mujeres viudas o aquellas que se encontraban en separaciones transitorias (por hospitalización, emigración e incluso, encarcelamiento...) eran consideradas familias a las que había que ayudar, puesto que la razón principal de dicha situación no era responsabilidad de las mujeres y por ello, contaban con una pensión económica pública. A continuación, se encontraban las mujeres separadas de forma definitiva 
que, a pesar del escándalo que generaban, también recibían por parte del estado una ayuda económica. Sin embargo, en el último puesto se encontraban las familias monoparentales de madres solteras, quienes además de no recibir ningún tipo de ayuda económica, sufrían un importante estigma social y fueron objeto durante mucho tiempo de rechazo.

La monomarentalidad, además, ha sido asociada durante mucho tiempo a la marginalidad, el consumo de drogas, la prostitución, la delincuencia y la crianza de hijas e hijos conflictivos. Ejemplo de ello es que en la literatura de los años cincuenta es muy común referirse al mismo como madres patológicamente desequilibradas (Finch y Summerfield, 1999).

La comprensión de la dinámica de la monomarentalidad y de los procesos de formación y disolución de los núcleos formados por un sólo progenitor, es esencial como acabamos de constatar históricamente para poder estimar la presencia y permanencia de este tipo de familias en el tiempo y para anticipar el trato social que podrán recibir y articular políticas sociales al respecto.

Las razones por las que una familia pasa a convertirse en monomarental son de carácter diverso: puede ser resultado de un acontecimiento vital estresante como una separación, divorcio o muerte del cónyuge, pero también el fruto de una elección libre y meditada. Las consecuencias, como es de esperar, no serán las mismas en uno u otro caso.

Actualmente los expertos en la materia identifican hasta 4 vías de acceso a la monomarentalidad: 1) maternidad en solitario o extra-conyugal, 2) maternidad en solitario por elección propia: madres adoptivas, 3) maternidad vinculada a la relación matrimonial previa: rupturas matrimoniales (separaciones o divorcio) y disoluciones de la familia por defunción, y 4) maternidad vinculada a situaciones sociales: emigración, trabajo, hospitalización o encarcelación. En cada una de estas vías de acceso, las mujeres configuran diferentes perfiles de maternidad y acompañan a sus hijos e hijas en situaciones diversas (Castro y Seiz, 2014; González Rodríguez, Jiménez, Morgano y Díez, 2004-2007; Flaquer, 2006; Perondi, 2012). A continuación se describe brevemente cada una de ellas:

1) Maternidad en solitario o extra-conyugal: Ser madre soltera consiste en tener descendencia sin un vínculo matrimonial. A pesar de que este término hace referencia a la opción de ser madre por decisión propia, este apartado se centra en describir mayoritariamente a mujeres que están en situación de embarazo no deseado y que han optado por asumir la maternidad en solitario (Perondi, 2012). Este es un factor de riesgo por lo que muchas mujeres si no cuentan con la ayuda tanto económica como afectiva de sus familiares se exponen a posibles situaciones de pobreza y/o exclusión social. Los datos señalan que, por lo general, son adolescentes en plena etapa de escolarización. Del total de los hogares monomarentales, este grupo representa el $13,1 \%$ en 2015 (INE, 2016).

2) Maternidad en solitario por elección propia: Madres adoptivas: Estas mujeres parten de situaciones más aventajadas en comparación con el resto de las familias 
monomarentales; son adultas, con buena situación económica, niveles educativos más altos, amplio abanico de redes sociales y amistades y, especialmente, surgen del deseo y la decisión propia de ser madres (González Rodríguez, Jiménez, Morgano, y Díez, 2004-2007).

3) Maternidad vinculada a la relación matrimonial previa: Rupturas matrimoniales (separación o divorcio) y disolución de la familia por defunción: Es una de las principales razones por las cuales la monomarentalidad ha crecido y se ha hecho más visible en los últimos años. A día de hoy es la principal vía de entrada a la monomarentalidad (INE, 2014).

A pesar de que en la mayoría de los países occidentales democráticos existía alguna forma de divorcio, hasta finales de los años 60 se trataba de regulaciones muy restrictivas y eran escasas las parejas casadas que optaban por esta vía. En España, el divorcio no fue aprobado hasta 1981 (Flaquer, Almeda y Navarro-Varas, 2006). En 2012 el 66,2\% de los casos en España que se divorciaron fueron de mutuo acuerdo; no obstante, en el caso de no ser así es más común que sea la mujer quien toma la iniciativa (Castro y Seiz, 2014).

A día de hoy, se observa cómo la viudedad va disminuyendo como flujo de entrada a la monomarentalidad. A principios del siglo XX la esperanza de vida al nacer en España se situaba en tan sólo 33,85 años en los varones y de 35,70 para las mujeres. No obstante, los avances registrados en nuestro país han multiplicado los años de esperanza de vida, situándola a finales del siglo XX y principios del siglo XXI en 75,25 años en los varones y en 82,16 años en las mujeres (Flaquer et al. 2006).

4) Maternidad vinculada a situaciones sociales: emigración, trabajo, hospitalización o encarcelación: Este tipo de familias tienen la particularidad de que pueden ser temporales/transitorias o provisionales, o instalarse en la permanencia y terminar en una separación o en divorcio con el paso de los años (Perondi, 2012); como puede ser en el caso de hospitalización pasando a la viudedad, o en el caso de encarcelación o emigración pasando a la ruptura de la pareja.

En suma, nos encontramos con cuatro vías de acceso a esta tipología familiar, siendo tres de ellas situaciones no escogidas, como es el caso de la maternidad en solitario o extra conyugal, la maternidad vinculada a la relación matrimonial previa y la maternidad vinculada a situaciones sociales como la emigración, el trabajo, la hospitalización o la encarcelación, mientras que, por otro lado, se encuentra la maternidad escogida, como son las madres adoptivas.

Desde una perspectiva de bienestar y riesgo de pobreza, la maternidad escogida se relacionaría con un mayor bienestar y niveles superiores de participación e inclusión social, debido a que el deseo de ser madre en solitario implica ser consciente de lo que supone tener un hijo y poder mantenerlo económicamente; siendo, por lo general, mujeres con un puesto de trabajo estable y un nivel de estudios superior a los de la media española. Mientras que la maternidad no escogida, se vincularía con una mayor vulnerabilidad y con un mayor riesgo de pobreza y exclusión social. 


\section{La familia monoparental en Europa, España y País Vasco}

El modelo de familia monomarental es cada vez más frecuente en nuestro entorno en consonancia con la realidad estatal e internacional y se observa un aumento gradual del número de este tipo de hogares junto a la eliminación de la imagen peyorativa a la que estaba sometida, ayudando así a su visibilización (Di Nella, Almeda y Ortiz, 2014; Giraldes, Penedo, Seco y Zubeldia, 1998).

En la tabla 2, se aportan datos descriptivos y de evolución sobre la realidad monoparental, en concreto del número y porcentaje de familias monoparentales en Europa, España y País Vasco, así como su evolución entre los años 2011 y 2016:

Tabla 2: Datos sobre las familias monoparentales en Europa, España y Comunidad Autónoma Vasca en los años 2011 y 2016

\begin{tabular}{lrrr|rrr}
\hline & $\begin{array}{r}\text { EUROP } \\
\text { A }\end{array}$ & ESPAÑA & CAPV & $\begin{array}{r}\text { EUROP } \\
\text { A }\end{array}$ & ESPAÑ & CAPV \\
\hline \multicolumn{1}{c}{ Año } & 2011 & 2011 & 2011 & 2016 & 2016 & 2016 \\
\hline $\begin{array}{l}\text { N. total } \\
\text { hogares }\end{array}$ & $\begin{array}{r}508.545 \\
227\end{array}$ & 18.083 .6 & 98.940 & & 18.406 .1 & 898.600 \\
\hline $\begin{array}{l}\text { N. hogares } \\
\text { monoparent } \\
\text { ales }\end{array}$ & 22.375 .9 & 361.673 & 39.677 & & -1.964 .90 & \\
\hline $\begin{array}{l}\text { \% hogares } \\
\text { monoparent } \\
\text { ales }\end{array}$ & $4,4 \%$ & $2,0 \%$ & $4,46 \%$ & & 0 & 94.100 \\
\hline
\end{tabular}

Fuente: Elaboración propia a partir de la información obtenida de Eurostat (2011), Gobierno Vasco (2014), INE (2011; 2016), Ikuspegi (2011) y Perondi (2012)

A nivel europeo, los últimos datos encontrados sobre familias monoparentales -sin especificar el género de la persona cuidadora- corresponden al 2011. Aparecen un total de 22.375.990 familias monoparentales en Europa, que corresponde al 4,4\% del total. Los países en los que estas familias se encuentran con más frecuencia son Noruega e Islandia, con más del $8 \%$ del total de estos hogares; seguidos por Dinamarca y el Reino Unido, con el 6,9\% y el 6,8\% respectivamente. En el extremo opuesto, se sitúan países como Polonia, Rumanía o Grecia que figuran en 2011 alrededor del 1,5\% del total de hogares (Eurostat, 2011; Perondi, 2012).

Desglosando una comparativa con otros países europeos, se observa que la figura de la familia monoparental-monomarental está menos desarrollada en España que en el resto de Europa. Así, en el año 2011 (INE, 2011), España cuenta con un total del 
$2,0 \%$ de familias monoparentales -sin especificar tampoco el género de la persona cuidadora- no obstante, este modelo se encuentra en alza en los últimos años en España. Como ejemplo de ello se observa que, en 2016, la Encuesta Continua de Hogares $(E C H)$ contabiliza un total de 1.964.900 familias monoparentales en España, lo que corresponde con un 10,86\% del total de hogares, de los cuales el 8,2\% están encabezados por mujeres (INE, 2016).

En el caso de la Comunidad Autónoma del País Vasco son 94.100 el número de familias monoparentales registradas en 2016 , constituyendo un 10,58\% del total de hogares, de los cuales el $8,6 \%$ se encuentran con la figura de la madre como referente de la prole. En la CAPV se observa también un importante crecimiento de estos hogares en términos absolutos puesto que en 2011 eran $39.677(4,46 \%)$ los hogares con un padre o una madre que convivían con algún hijo o hija menor dependiente (Gobierno Vasco, 2014; Ikuspegi, 2011; INE, 2016).

Un estudio realizado por el Eustat (2009) sobre la evolución de la estructura familiar en dos décadas (1986-2006) concluye que las familias monoparentales en la Comunidad Autónoma Vasca han alcanzado un porcentaje cercano al 9\%. La principal evolución que se observa se refiere a la vía de acceso a la misma, en donde aumentan el divorcio y la separación, disminuyendo la viudedad. En donde tampoco se advierten cambios significativos en estos últimos 20 años es en la distribución entre familias monomarentales, en torno al 85\% y las monoparentales, cercanas al $15 \%$.

Además de un acercamiento descriptivo, que nos permite constatar cómo el número de familias monomarentales va en aumento, se hace necesario un acercamiento a la caracterización y perfil general de estas familias y hogares.

A pesar de no contar ni con muchos estudios, ni con datos muy fiables, Perondi (2012) desarrolla un estudio relevante para el Instituto de la Mujer y el Fondo Social Europeo sobre las familias monoparentales (MP) y monomarentales (MM) a partir de la Encuesta de Población Activa (EPA) y de la Encuesta de Condiciones de Vida (ECV) desarrolladas en España, en los años 2011 (segundo trimestre) y 2010 respectivamente. La directora del informe, señala una serie de limitaciones metodológicas a tener en cuenta en los datos y análisis realizados que, desde nuestro punto de vista, no ponen en entredicho el valor de los mismos sino que los contextualizan y ponen en evidencia la dificultad de investigar en estos días sobre esta materia, puesto que dichas limitaciones se encuentran también en otros estudios:

- No diferenciar el sexo de la persona a cargo de la unidad familiar (MM o MP).

- No identificar la ruta o vía de acceso a la MM o MP.

- No tener en consideración otras variables como la edad, la comunidad autónoma de residencia, la condición de inmigrante o minoría étnica.

- Cuestiones de representatividad muestral (cuestión que incorpora en prácticamente casi todas las representaciones y tablas de su estudio).

La tabla 3 resume los escasos datos encontrados por Perondi en su estudio con estas dos fuentes y también recoge los pocos datos que añade el INE (2016). 
Tabla 3: Perfil de la Familia Monoparental/Monomarental según Encuesta de Población Activa - EPA (2011), Encuesta de Condiciones de Vida - ECV (2010) y Encuesta Continua de Hogares - ECH (2015)

\begin{tabular}{|c|c|c|c|}
\hline & & & \\
\hline \% POBLACIÓN & $\begin{array}{l}3,1 \% \text { hogares } \\
\text { españoles }\end{array}$ & $\begin{array}{l}2,6 \% \text { hogares } \\
\text { españoles }\end{array}$ & $\begin{array}{l}10,3 \% \text { hogares } \\
\text { españoles }\end{array}$ \\
\hline SEXo & $85,15 \%$ Mujeres & $80,1 \%$ Mujeres & $81,3 \%$ Mujeres \\
\hline EDAD MEDIA & 43 años & 44 años & \\
\hline $\begin{array}{l}\text { ORIGEN/ } \\
\text { NACIONALIDAD }\end{array}$ & 80\% Españolas & 91\% Españolas & \\
\hline $\begin{array}{l}\text { SITUACIÓN } \\
\text { CIVIL }\end{array}$ & $56,6 \%$ sep./div. & $54,3 \%$ div./sep. & $\begin{array}{l}36,4 \text { sep./div. } \\
38,15 \text { viuda }\end{array}$ \\
\hline $\begin{array}{l}\text { Nivel } \\
\text { EDUCATIVO }\end{array}$ & $\begin{array}{l}32,1 \% \text { Univ. FP sup. } \\
\text { y doct. } \\
27,2 \% \text { Est. sec. y FP }\end{array}$ & $\begin{array}{l}26,1 \% \text { Univ. FP sup. } \\
\text { y doct. } \\
28,8 \% \text { Est. sec. y FP }\end{array}$ & \\
\hline $\begin{array}{l}\text { SITUACIÓN } \\
\text { LABORAL }\end{array}$ & $\begin{array}{l}66 \% \text { ocupadas } \\
21,3 \% \text { desempleo } \\
12,7 \% \text { inactivas } \\
\end{array}$ & $\begin{array}{l}63,8 \% \text { ocupadas } \\
22,9 \% \text { paradas } \\
13,3 \% \text { inactivas }\end{array}$ & \\
\hline HIJOS E HIJAS & $\begin{array}{l}1 \text { ó } 2 \text { menores a } \\
\text { cargo } \\
\text { MM 1,55>MP 1,42 a } \\
\text { cargo } \\
\text { MM 65,5\%>MP } \\
48,6 \%>16\end{array}$ & $\begin{array}{l}1,52 \text { hijos/as a cargo } \\
\mathrm{MM}_{1,59}>\mathrm{MP}_{1,26} \\
\text { a cargo }\end{array}$ & 1 hijo/a a cargo \\
\hline
\end{tabular}

Fuente: Elaboración propia a partir de Perondi (2012) e INE (2016)

Esta tabla nos permite observar varias cuestiones importantes con respecto al perfil y caracterización global de este tipo de familias. En primer lugar, en relación a la evolución cuantitativa, los escasos datos hallados apuntan, sin embargo, de manera consistente hacia un aumento en los últimos años que alcanza un 10\% del total de hogares de la población en general. La monoparentalidad creció un 8,1\% de media, pero más entre los padres que entre las madres. Es decir, creció más la monoparentalidad (en un 16,9\%) que la monomarentalidad (en un 6,3\%) (INE, 2016).

En segundo lugar, y en relación a las variables sociodemográficas, las mujeres encabezan la mayoría de estos núcleos familiares. En el caso de la EPA (2011) apunta al $85 \%$ mientras que la ECV (2010) señala el $80 \%$, discrepancias posibles tanto por la fuente como por el año de estudio. También parece haber una coherencia en destacar la separación y el divorcio como las vías de acceso a esta tipología familiar. Por otro lado, INE (2016) también esboza las vías de acceso más comunes a la monomarentalidad en España, encontrando que el 36,4\% de las mujeres están separadas o divorciadas, seguido de las mujeres viudas con un $35,8 \%$, y con un $13,1 \%$ las solteras, donde se encuentra la maternidad elegida. Perondi (2012) en su estudio señala que la edad promedio de la madre en España en estas familias se encuentra 
entre los 45 y 55 años. A esta investigación añade el INE (2016) que un $65 \%$ de las mujeres tienen hasta 65 años de edad, lo que coincide también en que la mayoría de las mujeres se encuentran entre estos rangos de edad. No obstante, en el País Vasco, la edad disminuye, situando a la madre del hogar monomarental entre los 39 y 64 años de edad (Ikuspegi, 2010). En relación a la nacionalidad de estas mujeres, según los datos ofrecidos por la Encuesta de Población Activa del INE (2016) el 79,8\% tienen nacionalidad española. Asimismo, las zonas con mayor porcentaje de este tipo de familias son Andalucía con un porcentaje de 19,3\%, Cataluña con un 16,6\%, Navarra y Cantabria con un 1,2\% y La Rioja con un 0,6\%.

En tercer lugar, en lo que se refiere al nivel educativo y la situación laboral, llama la atención el nivel educativo alcanzado y el nivel de ocupación. No se pueden olvidar dos cuestiones en la interpretación de este dato: Por una parte, que en los datos generales se encuentran recogidas todas las vías de acceso a la monomarentalidad, incluida también la opción libre de las madres adoptivas. Por otra parte, es también importante tener en cuenta la edad media de estos hogares y recordar los niveles de acceso al sistema educativo como derecho universal. De hecho, donde mayores diferencias se observan es en los niveles primarios entre las familias monoparentales / monomarentales y la población general.

\section{La familia monomarental: la exclusión en la opción "no elegida"}

Una mirada al pasado es sin duda la vía adecuada para encontrar claves sobre cómo se ha ido construyendo en nuestro entorno el modelo de integración social y de exclusión, y cómo se ha ido transformando hasta nuestros días; sólo así se pueden entender los límites que este modelo de integración muestra. Jiménez (2008, p.177) describe la zona de exclusión como aquella que "se construye desde la inestabilidad en el empleo, la renta y la desprotección, pero conformada como expulsión y no simplemente como precarización o carencia, y a la vez se combina con el aislamiento social".

Aun manteniendo que la exclusión social es un proceso cambiante, es necesario analizar su heterogeneidad. Repasando los diversos colectivos y/o grupos que pueden tener una especial relevancia dentro de la exclusión, se encuentra la monomarentalidad no escogida, que pone de realce la perspectiva de género en la exclusión social (Alcalde Campo y Pávez, 2013; Laparra y Pérez, 2008; Lupica, 2012; Sastre, 2015).

A la hora de referirse al binomio monomarentalidad y exclusión social, indefectiblemente se debe aludir al concepto de feminización de la pobreza. Este término, desarrollado por Pearce en 1978, describe un conjunto de situaciones con mayor presencia, intensidad y riesgo de pobreza entre las mujeres en comparación con los hombres. Es decir, concibe a las mujeres como un grupo especialmente vulnerable y con mayor riesgo de pobreza (Canto, Mier, Uribelarrea, Acha y Barbero, 
2004; Carrasco, 2014; Damonti, 2014; Fundación FOESSA, 2008; Fuller, 1999). Alter (2008) y Geldstein (1997) añaden que el aumento de la pobreza y/o exclusión social se encuentra en relación directa con el aumento de la pobreza de los hogares monomarentales, dado que éstos son un grupo diana para la pobreza, refiriéndose sobre todo a la monomarentalidad no elegida.

Algunas de las primeras investigaciones que incorporan este concepto se realizaron entre los años 80 y 90 en Estados Unidos, extrayendo como consecuencia que a medida que la pobreza intrafamiliar aumentaba, también se incrementaba el riesgo de pobreza en los hogares monomarentales. En efecto, la separación, el divorcio y la viudedad suponen para muchas madres la pérdida del estatus económico, social y personal, lo cual genera una inestabilidad inherente que acrecienta el riesgo de pobreza (Alter, 2008).

Las causas que conllevan a los hogares monoparentales y monomarentales a la exclusión social, el tipo de situaciones vividas y los mecanismos para salir de ella son altamente diferenciables por género. Mientras que las causas identificadas en los hombres tienen que ver con el desempleo, la discapacidad o las drogodependencias, en las familias monomarentales se identifican como factores de riesgo los problemas relacionados con sus capacidades como reproductoras (madres solteras), rupturas afectivas (fallecimiento del cónyuge, separaciones, divorcios o malos tratos), rupturas por problemas sociales (encarcelamiento de la pareja/maridos), sobrecarga de tareas en su rol como madre y cuidadora de la familia o su mayor fragilidad en el mercado laboral; en términos generales, problemas procedentes de su especialización en la reproducción doméstica y social y por su consecuente dependencia afectivaeconómica (Alter, 2008; Laparra y Pérez, 2008).

Estas causas son confirmadas por dos estudios desarrollados previamente en esta materia (IDD, 2015; González de Audikana y Aróstegi, 2017). En concreto, González de Audikana y Aróstegi (2017) señalan cómo cuando las mujeres se encuentran inmersas en procesos de exclusión se produce la denominada doble discriminación, que se relaciona directamente con una sociedad patriarcal en donde las mujeres se dedican no sólo a la reproducción sino también a la crianza y cuidado de la prole y la familia. Estas tareas, consideradas socialmente como "sagradas" generan ciertas expectativas, de manera que la mujer que no atiende dichas tareas bajo tal criterio, se convierte en una "mala mujer", "una mala madre" o "una mala esposa". Como consecuencia aparecen dos manifestaciones: una primera más visible y material se refiere a que las mujeres han accedido a posiciones subordinadas en los trabajos productivos, fuera del hogar, cuentan con menor cualificación, peores empleos, peores condiciones laborables y menor sueldo, en comparación con los hombres. La segunda manifestación se refiere al estigma social. Cuando una mujer no cumple con las expectativas sociales establecidas, cuenta con un estigma mucho peor que el del varón en circunstancias semejantes. Es decir, el estigma social de una mujer encarcelada o de una mujer adicta es mayor que el recibido por el varón. Además, 
dicho estudio (González de Audikana y Aróstegi, 2017) pone de manifiesto el proceso por el cual las mujeres interiorizan el rol, la expectativa y el estigma asociado a su incumplimiento hasta convertirlo en auto-reproche y culpa. Estos problemas se verán agudizados si la mujer tiene personas ancianas o dependientes a su cargo, pero especialmente si son hijos y/o hijas, de manera que, ante el temor de que le retiren la custodia y también tratando de evitar su sufrimiento, ocultará su situación con el consiguiente agravamiento.

Una parte importante de la caracterización de los hogares en exclusión viene dada por el perfil de la persona principal sustentadora a través de factores de riesgo como el género, la edad, el lugar de nacimiento y el estado civil, junto a otros como el nivel de estudios y la relación con la actividad económica, la vivienda y la salud, o las redes de apoyo. A través de estas variables, se analiza y describe el perfil de la familia monomarental y su incidencia mayor o menor en el riesgo de exclusión social o pobreza.

1) Género y edad: En cuanto al género se observa que la inmensa mayoría tiene rostro de mujer y que las cifras se encuentran en torno al $80 / 85 \%$.

La mayoría de los hogares son, por tanto, monomarentales. Las razones por las que las mujeres acceden a este tipo de hogares se deben como hemos visto a viudedad, separación, divorcio, conflictos conyugales o malos tratos principalmente, es decir, rupturas en donde existe dependencia económica, mientras que en el $18 \%$ o $20 \%$ de los hombres restantes por dificultades de empleo, discapacidad y drogodependencias (Laparra y Pérez, 2011). En otras palabras, las diferencias son en términos absolutos y en vías de acceso, y según los expertos, los hogares encabezados por mujeres son especialmente vulnerables a la exclusión social aunque no lleguen a situaciones extremas porque utilizan otras estrategias de compensación. Del total de familias monomarentales el riesgo de pobreza y exclusión ha aumentado 6 puntos en el 2014 respecto al 2013, alcanzando ya un total de 53,3\%, según el Informe de Save the Children (Sastre, 2015). A esta búsqueda de datos sobre el perfil de la madre en exclusión Adecco (2016) añade que el rango de edad de la mujer sustentadora del hogar oscila entre los 36 y 45 años de edad, encontrando en esta franja los mayores obstáculos en su modelo de integración y bienestar; consecuencia en la mayoría de los casos $(56,6 \%)$ de una ruptura con la pareja y la dependencia económica que se amparaba (Perondi, 2012).

2) Lugar de nacimiento y estado civil: El hecho de ser originario/a de otro país representa un factor de especial vulnerabilidad, debido a las dificultades de adaptación que pueden encontrar respecto a su red social de apoyo, junto a otras variables como el aprendizaje lingüístico y los códigos culturales (Laparra y Pérez, 2008). Así, nos encontramos con que un $20,2 \%$ del total de familias monomarentales en España son extranjeras (Perondi, 2012).

En relación a la presencia de familias monoparentales / monomarentales en riesgo de pobreza y, siguiendo con los datos aportados por la Fundación Adecco (2016), 
encontramos a Andalucía $(35,7 \%)$ y Murcia $(31,8 \%)$ con un mayor número de pobreza en este tipo de hogares, mientras que Navarra $(9,6 \%)$ y País Vasco $(10,6 \%)$ son las regiones con menor ocurrencia de pobreza entre los hogares monomarentales.

Por otro lado, aludiendo al estado civil que se encuentra con mayor riesgo de pobreza dentro de la monomarentalidad son las madres divorciadas o separadas $(56,6 \%)$ seguidas de las solteras $(21,2 \%)$ y las adolescentes (Perondi, 2012). Lo reflejado anteriormente indica que las madres divorciadas o separadas encuentran mayores dificultades económicas para respaldar a sus propios hijos, debido a la dependencia afectiva-económica de su antigua pareja.

3) Nivel de estudios y actividad económica: En tercer lugar, es importante indagar sobre el nivel de estudios y la actividad económica principal de quien sustenta la familia en exclusión, la madre. Los estudios y el empleo se vuelven así un aspecto clave para entender e interpretar la exclusión social. De esta forma se comprueba que la mayor inestabilidad de la exclusión en el hogar es reflejada en una relación más precaria de la figura que sustenta con el mercado de trabajo.

A rasgos generales, Alter (2008) determina que los hogares monomarentales se caracterizan por tener una baja cualificación tanto educativa (estudios reglados) como laboral (baja o falta de cualificación) acompañada de una ausencia de historia laboral previa que facilite la incorporación en el mercado laboral. Junto a Alter (2008), estudios más actuales realizados tras la crisis como el de Damonti (2014) y el de Save the Children (Sastre, 2015) señalan cómo el trabajo de estas mujeres se caracteriza por ser precario e inestable, discontinuo y a tiempo parcial, y en consecuencia, con salarios inferiores respecto a los hombres. Alter (2008) especifica que los trabajos más comunes desarrollados por estas mujeres son en el ámbito doméstico, la limpieza y la hostelería.

No obstante, la Fundación Adecco (2016) señala que 7 de cada 10 lleva más de un año sin trabajo, un $18 \%$ está en búsqueda de su primer empleo, y un $53 \%$ se encuentran desempleadas. A lo que añade que "la pobreza infantil cuando la madre está en paro afecta a tres de cada cuatro niños, pero sorprende negativamente que aunque trabaje, más de la mitad de los niños siguen en situación de pobreza" (Sastre, 2015, p.11), lo que significa que la pobreza no sólo afecta a las áreas económicas sino que también afecta tanto a los aspectos relacionales, educativos como políticos.

4) Situación del hogar / vivienda y salud: En cuarto lugar, otra de las variables para determinar un perfil específico de la monomarentalidad en riesgo de pobreza es la situación del hogar (tipo de vivienda) y la salud.

Respecto al tipo de vivienda en el que viven las familias monomarentales en exclusión puede extraerse que están construidas por pocos metros cuadrados o incluso en condiciones de no habitabilidad (sin calefacción, sin agua caliente, con una única habitación...). Un 53\% de quienes viven en hogares monomarentales se ven afectados por problemas que responden a situaciones de exclusión en la vivienda y la mayoría viven en barrios marginales siguiendo a Save the Children (Sastre, 2015). 
La Fundación Adecco (2016) añade que la falta de recursos para poder hacerse cargo de los pagos de la casa o el alquiler, condiciona que aproximadamente el $40 \%$ comparta vivienda con sus padres $u$ otros familiares/cercanos, si la relación afectiva con los mismos lo permite, ya que no todas las familias poseen una red de familia o amigos estables.

Por otra parte, no disponer de ingresos económicos ha conllevado en múltiples hogares a la eliminación de dietas nutritivas en los/as hijos/as y sus madres, hecho que debilita -sobre todo- el desarrollo del menor. Reducir gastos en la alimentación aumenta el riesgo de padecer problemas de salud. De igual manera, la falta de recursos supone también una eliminación de medicamentos necesarios para seguir un tratamiento por enfermedad o discapacidad.

Finalmente, Gumá, Treviño y Cámara (2015) estudian la relación entre la posición en el hogar y la calidad de vida relacionada con la salud (CVRS) entre la población de 30 a 64 años en España, llegando a la conclusión de que la monomarentalidad es la posición del hogar asociada a una peor CVRS, por su carga continua de ansiedad y depresión, mayoritariamente. En suma, Alter (2008) y Martínez (2014) amplían esta hipótesis señalando que las razones principales por las que acuden a los servicios médicos, tanto las madres como los/as hijos/as e hijas de familias monomarentales suelen ser depresiones, ansiedad, o desnutrición.

5) Redes de apoyo: Alter (2008), Damonti (20014), Perondi (2012) y Save the Children (Sastre, 2015) coinciden en señalar que la falta de redes de apoyo es otra de las características que describe la situación de hogares monomarentales en situación de pobreza o riesgo de pobreza. Éstos se caracterizan por la falta de redes sólidas, al no disponer de tiempo para conciliar su vida laboral, familiar y social. Por lo que muchas mujeres terminan perdiendo relación con su grupo de iguales e incluso con su familia.

6) Riesgo y tipo de pobreza: La situación de vulnerabilidad y pobreza es otra variable importante para analizar dentro de las familias monomarentales, con el fin de conocer con más exactitud el fenómeno al que están sometidos más de la mitad de estos hogares. Save the Children en su Informe Más solas que nunca (Sastre, 2015) detalla que más de la mitad de estos hogares se encuentran en riesgo de pobreza. La pobreza moderada alcanza ya el $40 \%$ de los hogares monomarentales en 2014 y la pobreza severa el 14\%. La pobreza moderada hace referencia al inicio del proceso de exclusión y, se traduce en hogares con un impacto de exclusión superior al doble de la media, mientras que, la pobreza severa acompaña los procesos de exclusión y es más difícil salir de esta situación (Villafranca, Ramírez del Río y Gómez, 2014).

Los datos de la Encuesta de Pobreza y Desigualdades Sociales (EPDS) permiten observar que en la CAPV un $34,3 \%$ de las familias monomarentales se encuentran en riesgo de precariedad en 2010. Esto indica que, a pesar de que la tasa de familias monomarentales en riesgo de pobreza sea menor que en España, es uno de los colectivos más vulnerables de sufrir situaciones de exclusión (Ikuspegi, 2010). 
El hecho de que los porcentajes de riesgo de pobreza y exclusión sean más elevados que los del conjunto de la población se debe a que la figura de la madre no sólo se responsabiliza por partida doble en calidad de proveedora del sustento y cuidado de la familia, sino también a una falta de apoyo y de red para sostener esta situación, con el resultante deterioro emocional y dificultad para adecuar la vida familiar y laboral (Sastre, 2015).

\section{Conclusiones}

En este artículo se ha realizado un análisis de la monoparentalidad monomarentalidad y su relación con la exclusión social, analizando cómo su definición y su visión ha evolucionado en el transcurso del tiempo y las implicaciones que esto ha supuesto y los riesgos que aún hoy amenazan estos modelos de familia.

Las familias monoparentales y monomarentales están constituidas por un padre o una madre a cargo de al menos un hijo o hija menor de 18 años. Es a finales del siglo $X X$ cuando en España se ha ido ampliando el estudio sobre las familias monoparentales, y a principios del siglo XXI, cuando se recategoriza a estas familias en términos femeninos, bajo el nombre de familias monomarentales, al observar que la mayoría de las familias están representadas por la figura de la madre como cuidadora de su prole.

Las vías de entrada o acceso a la monomarentalidad son: 1) maternidad en solitario o extra-conyugal; 2) maternidad en solitario por elección propia; 3) maternidad vinculada a la relación matrimonial previa: rupturas matrimoniales (separación o divorcio) y disolución de la familia por defunción; 4) maternidad vinculada a situaciones sociales: emigración, trabajo, hospitalización o encarcelación. Y su impacto es diferencial a la hora de padecer un mayor o menor riesgo de exclusión, intensificándose en las vías denominadas "no elegidas".

Se han presentado cuatro vías de acceso a esta tipología familiar, siendo tres de ellas situaciones no escogidas, como es el caso de la maternidad en solitario o extra conyugal, la maternidad vinculada a la relación matrimonial previa y la maternidad vinculada a situaciones sociales como la emigración, el trabajo, la hospitalización o la encarcelación, mientras que, por otro lado, se encuentra la maternidad escogida, como son las madres adoptivas.

La evolución cuantitativa de las familias monoparentales sigue una tendencia creciente tanto en Europa, España como CAV situándose en torno al 10\% de las familias y de forma paralela son muchas las familias dentro de esta tipología que se encuentran en situación de alta vulnerabilidad o exclusión social. Los factores de riesgo que se han presentado giran en torno a los siguientes ejes: 1) Género y edad, 2) Lugar de nacimiento y estado civil, 3) Nivel de estudios y actividad económica, 4) Situación del hogar / vivienda y salud, 5) Redes de apoyo. En función de estos ejes se configura el 6) Riesgo y tipo de pobreza. 
De manera conclusiva se concibe que llevar a cabo la monomarentalidad en solitario aumenta el riesgo de pobreza; es como si fuera un bucle del que es difícil salir configurado por dos parámetros: la monomarentalidad en solitario lleva rápidamente a la pobreza y la pobreza en solitario aumenta su grado y su intensidad, lo que lleva también a la transmisión intergeneracional de la pobreza. A diferencia de la maternidad escogida, la familia monomarental no elegida se correlaciona estrechamente con los procesos de exclusión social y pobreza, afectando a un $53 \%$ del total. La persona responsable de estos hogares es una mujer de 30 a 45 años, aproximadamente, de nacionalidad española $y$, con problemas de desempleo laboral y trabajos inestables. Tienen problemas de ansiedad y depresión $y$, la vivienda y alimentación han perdido importancia en su vida: viven en casas inhabilitadas de escasos metros cuadrados, comparten vivienda con otras personas y, para poder pagar el alquiler ahorran en alimentación, eliminando dietas nutritivas. Así es como viven más de la mitad de estos hogares ( $53 \%$ en 2015 ).

La realidad creciente de estas familias junto a las necesidades vinculadas a las mismas constituye una llamada a la acción e intervención social y psicoeducativa en aras de disminuir el riesgo de pobreza, aumentar su bienestar social y trabajar por una sociedad más inclusiva que encuentra espacios y lugares para todas las personas.

\section{Bibliografía}

Alcalde Campo, R., y Pávez, I. (2013). Infancia, familias monoparentales e inmigración latinoamericana en Barcelona, España. Revista Latinoamericana de Ciencias Sociales, Niñez y Juventud, 11 (1), 229-243.

Alter - Grupo de investigación. (Julio de 2008). Monoparentalidad y Exclusión Social. Obtenido de II Plan de Lucha contra la Exclusión Social en Navarra. Universidad de Navarra: Departamento de trabajo social.

Andolfi, M. (1993). Terapia Familiar. Un enfoque interaccional. Barcelona: Herder.

Avilés, M. (2012). Origen del concepto de monoparentalidad. Un ejercicio de contextualización sociohistórica. Papers Revista de Sociología, 98 (2), 263-285.

Avilés, M. (2015). ¿Qué es la monoparentalidad? Una revisión crítica de su conceptualización en materia de política social. Cuadernos de Trabajo Social, 28 (2), 211-223.

Barrón, S. (2002). Familias monoparentales: un ejercicio de clarificación conceptual y sociológica. Revista del Ministerio de Trabajo y Asuntos Sociales, 40, 13-30.

Canto, A., Mier, I., Uribelarrea, E., Acha, N., y Barbero, I. (2004). Los procesos de Exclusión Social de las mujeres solas en la CAPV. Vitoria: Fundación Ede.

Carrasco, L. (2014). Mujeres sin hogar en España: Un análisis sociográfico desde una perspectiva feminista. Universidad Complutense, España.

Castro, T., y Seiz, M. (2014). La transformación de las familias en España desde una perspectiva socio-demográfica. Fundación Foessa, (4).

Damonti, P. (2014). Una mirada de género a la exclusión social. Fundación Foessa, (4).

De Lourdes, L. (2003). Dinámica de la familia. Un enfoque psicológico sistemático. México D.C: Pax México. 
Di Nella, D., Almeda, E., y Ortiz, R. (2014). Perspectiva no androcéntrica en los estudios sobre familias monoparentales. Reflexiones e implicaciones metodológicas. Athenea Digital (14), 181-207.

EUROSTAT. (2011). Geographical area and family status. Eurostat. Recuperado de: http://ec.europa.eu/eurostat/statistics-

explained/index.php/People in the_EU_\%E2\%80\%93_statistics on household_and family structures.

EUSTAT (2009). Evolución Estructura Familiar. Análisis de resultados. 2006. VitoriaGazteiz. Recuperado

de: http://www.eustat.eus/elementos/ele0005000/ti_Evolucion_Estructura_Famili ar_2006_Analisis_de_resultados_pdf_616_KB/info005051_c.pdf

Finch, J., y Summerfield, P. (1999). Social Reconstruction and the Emergence of Companionate Marriage 1945-1959. Oxford: Blackwell Publishers.

Flaquer, L., Almeda, E., y Navarro-Varas, L. (2006). Monoparentalidad e Infancia. Colección Estudios Sociales, (20).

Fuller, N. (1999). ¿Pobreza o desigualdad de género? El caso de las familias jefaturadas por mujeres. Revista UM FDER, 10, 43-60.

Fundación Adecco. (2016). V Informe de la monoparentalidad y empleo. Madrid.

Fundación Foessa. (2008). Género y exclusión social en la monoparentalidad. Navarra: Fernández, B. y Iturbide, R.

Geldstein, R. (1997). Mujeres jefas del hogar: familia, pobreza y género. Centro de Estudios de Población. Argentina: UNICEF.

Giraldes, M., Penedo, E., Seco, M., y Zubeldia, U. (1998). La familia monoparental. Dialnet, 17-39.

Gobierno Vasco. (2014). Comunidad Autónoma de Euskadi. Encuesta de Necesidades Sociales 2014 Módulo EPDS-Pobreza. Comunidad Autónoma del País Vasco: Órgano Estadístico Específico del Departamento de Empleo y Políticas Sociales.

González de Audikana, J.M. y Aróstegi, E. (Dirs.) (2017). Mujeres en situación o riesgo de exclusión social que no acceden a los sistemas de protección social. Investigación Bizkailab no publicada. Universidad de Deusto.

González Rodríguez, M., Jiménez, I., Morgano, B., y Díez, M. (2004-2007). Madres solas por elección. Análisis de la monoparentalidad emergente. Estudios e Investigación Instituto de la Mujer, 53.

Gumá, J., Treviño, R., y Cámara, A. (2015). Posición en el hogar y género. Desigualdades en la calidad de vida relacionada con la salud entre la población adulta en España. Revista Internacional de Sociología, 73 (1), 1-17.

IDD - Instituto Deusto Drogodependencias (2015). Procesos de exclusión social femenina y análisis de las diversas barreras en su itinerario de inclusión. Investigación Bizkailab no publicada. Universidad de Deusto.

Ikuspegi (Diciembre de 2010). Análisis de los programas de conciliación orientados a las familias monoparentales en el País Vasco. Gizarte Gaien Behatokiak. http://ikuspegi-infancia.siis.net/en/investigacion/ver-estudio/383/

Ikuspegi. (2011). La situación de las familias en Euskadi. Obtenido de Departamento de empleo y asuntos sociales. Gizarte Gaien Behatokiak. http://ikuspegifamilia.siis.net/eu/investigacion/ver-estudio/377/ 
INE (2016). Tipos de hogar más frecuentes. Obtenido de Encuesta Continua de Hogares ECH. Recuperado de: http://www.ine.es/jaxi/Tabla.htm?path=/t20/p274/serie/def/po3/lo/\&file=03003 $. p \times \& L=0$

INE (2014) Boletín informativo del Instituto Nacional de Estadística. Las formas de la convivencia. Recuperado de: http://www.ine.es/ss/Satellite?L=es_ES\&c=INECifrasINE_C\&cid=12599444078 96\& $=1254735116567 \&$ pagename=ProductosYServicios\%2FPYSLayout

INE (2011). Censo de población y viviendas. Recuperado de: http://www.ine.es/censos2011_datos/cen11_datos_inicio.htm

Jiménez, M. (2008). Aproximación teórica de la exclusión social: Complejidad e impresión del término. Consecuencias para el ámbito educativo. Estudios Pedagógicos XXXIV (1), 173-186.

Laparra, M. y Pérez, B. (2008). La exclusión social en España: un espacio diverso y disperso en intensa transformación. Revista Sociológica, 6, 175-293.

Lorence, B., Pérez, J., Menéndez, S., y Rodríguez, I. (2015). Diversidad familiar: Una experiencia docente en el grado de trabajo social. Cuadernos de Trabajo Social, 22, 33-51.

Lupica, C. (2012). Madres solas: ¿Más vulnerables a la pobreza? Observatorio de la Maternidad, (51).

Martínez, L. (2014). Síntomas de agotamiento de la solidaridad familiar. Informe sobre exclusión y desarrollo social en España 2014. Madrid: Fundación Foessa.

Martínez-Monteagudo, M.-C., Estévez, E., y Inglés, C. (2013). Diversidad familiar y ajuste psicosocial en la sociedad actual. Revista Internacional de Psicología: Psicología.com, 17 (6), 1-22.

Perondi, A. C. (Dir.) (2012). Familias formadas por una sola persona adulta con hijo(s) y/o hija(s) a su cargo: diagnóstico y propuestas. Madrid: Centro de Estudios Económicos Tomillo, S.A.

Rodríguez, C., y Luengo, T. (2003). Un análisis del concepto de familia monoparental a partir de una investigación sobre núcleos familias monoparentales. Papers Revista de Sociología (69), 59-82.

Samaranch, E., y Di Nella, D. (2011). Introducción hacia un enfoque integral de la monoparentalidad. Familias monoparentales, violencias, discriminaciones y vulnerabilidad. Colección Familias monoparentales y diversidad familiar, 5 (11), 125-141.

Santibáñez, R., Flores, N. y Martín, A. (2017). Mujer en exclusión, Infancia en exclusión. Investigación Bizkailab no publicada. Universidad de Deusto.

Sastre, A. (2015). Más solas que nunca. La pobreza infantil en familias monomarentales. País Vasco: Save the Children España. Recuperado de: www.savethechildren.es/publicaciones/mas-solas-que-nunca

Sastre, A. y Escorial, A. (2017). Desheredados. Anexo Euskadi. Desigualdad infantil, igualdad de oportunidades y políticas públicas en España. País Vasco: Save the Children España. Recuperado de: www.savethechildren.es/publicaciones/desheredados

Song, M. (1996). Changing conceptualizations of lone parenthood in Britain: Lone parents or single mums? European Journal of Women's Studies (3), 377-397. 
UNICEF. (2003). Nuevas formas de familia: Perspectivas nacionales e internacionales. Uruguay: Udelar.

Valdivia, C. (2008). La familia: concepto, cambios y nuevos modelos. Revista Deusto Universidad, 1, 15-22. Universidad de Deusto.

Villafranca, R., Ramírez del Rio, A., y Gómez González, E. (2014). El perfil sociológico de la exclusión en España y su evolución. Madrid: Fundación Foessa.

Cómo referenciar este artículo/How to reference this article:

Santibánez, R.; Flores, N.; Martin, A. (2018). Familia monomarental y riesgo de exclusión social. iQUAL. Revista de Género e Igualdad, 1,123-144, doi: 10.6018/iQual.307701

Santibánez, R.; Flores, N.; Martin, A. (2018). Familia monomarental y riesgo de exclusión social. [Monomarental family and risk of social exclusion]. iQUAL. Revista de Género e Igualdad, 1, 123-144, doi: 10.6018/iQual.307701 